ISSN 0001-6002/2011/53/3/144-148 Acta Médica Costarricense, (C2011 Colegio de Médicos y Cirujanos

\title{
Original
}

\section{Bronquiectasias, estudio de 36 pacientes intervenidos en el hospital "Dr. Rafael Ángel Calderón Guardia"}

\author{
(Bronchiectasis, Results of Surgical Intervention in 36 \\ Patients)
}

José Alberto Mainieri-Hidalgo

\section{Resumen}

Objetivo: Analizar el resultado de la cirugía por bronquiectasias en el Servicio de Cirugía de Tórax del Hospital Calderón Guardia.

Método: Se revisaron los expedientes clínicos de 36 pacientes operados, con un total de 44 intervenciones por bronquiectasias.

Resultados: Se encontró una prevalencia muy significativa de la enfermedad en mujeres, con una relación de 11 a 1 . La etiología fue en 10 casos el antecedente de tuberculosis, en uno el síndrome de Kartagener, y en 25 no se logró establecer. Todos los pacientes tenían antecedente de tos y expectoración mucopurulenta de larga evolución, y 29 habían sido clasificados como asmáticos, aunque 10 no tenían ningún antecedente familiar. La radiografía de tórax fue anormal en todos los casos, mostrando áreas de fibrosis, infiltrados o nódulos, pero no fue útil para establecer el diagnóstico de bronquiectasias, contrario a la TAC, que en todos mostró las lesiones bronquiales. El resultado de la cirugía, contrario a lo esperado, no logró establecer un indicador de éxito, pues pacientes con lesiones muy localizadas continuaron expectorando e infectándose, y otros con patología difusa y bilateral, mejoraron sustancialmente.

Conclusiones: La cirugía por bronquiectasias está indicada cuando el tratamiento médico no logra controlar satisfactoriamente los cuadros infecciosos bronquiales. Existe franca posibilidad de mejoría, pero no un indicador de éxito para el procedimiento quirúrgico.

Descriptores: Bronquiectasias, enfermedad pulmonar obstructiva crónica, EPOC

\section{Abstract}

Aim: To analyze the results of surgical interventions due to bronchiectasias at the Thoracic Surgery Department of the Dr. R. A. Calderón Guardia Hospital.

Servicio de Cirugía de Tórax Hospital Dr.R.Á. Calderón Guardia Abreviaturas: HCG, Hospital Dr. Rafael Ángel Calderón Guardia; TAC, Tomografía Axial Computarizada; $\quad$ EPOC, Enfermedad pulmonar obstructiva crónica Correspondencia: José Alberto Mainieri Hidalgo mainierijose@hotmail.com
Methods: In order to review the results of 44 surgical interventions due to bronchiectasias at the Thoracic Surgery Department of Calderón Guardia Hospital, the clinical files of 36 patients were examined.

Results: A significant prevalence of the disease in female patients was observed, in proportion of 11 to 1 . In most cases, causal determination was not established due to lack of documentation in the clinical history annotations. In 10 cases there was a history of tuberculosis. One case corresponded to a Kartagener syndrome as causal elements of the disease. All patients exhibited 
a history of coughing and long evolution of mucopurulent expectoration. Twenty nive patients were catalogued as asthmatic even though ten of them lacked a family history of such disease. Thoracic X-Ray results were abnormal in all cases, presenting fibrosis, infiltrates or nodules, but were insufficient to provide diagnosis. CT scan results, in comparison, were effective in demonstrating the presence of bronchiectasias in all cases. Surgical intervention was decided upon when the affected area was localized in a specific part of the lung or when infectious processes became frequent and too difficult to control with medical treatment.

Conclusion: Results of surgery demonstrated that there was no effective indicator for success rates; some patients with particularly localized lesions continued to suffer from infection and expectoration, while others with diffuse and bilateral pathology demonstrated substantial improvements.

Key words: Bronchiectasias, Chronic obstructive pulmonary disease, COPD

Recibido: 21 de marzo de 2011

Aceptado: 5 de mayo de 2011

Las bronquiectasias son dilataciones anormales e irreversibles de los bronquios, adquiridas como secuela de procesos inflamatorios agudos o crónicos. Fueron descritas por Laennec en 1819, como un proceso supurativo con dilataciones bronquiales, y posteriormente clasificadas por Reid, en 1950, como cilíndricas, varicosas y saculares o quísticas. ${ }^{1,2} \mathrm{La}$ dilatación se acompaña de alteraciones del epitelio y de las glándulas bronquiales. Esto se traduce en un aumento en la producción de secreciones que se acumulan, se infectan y dan lugar al cuadro clínico de tos productiva y fiebre, pudiendo llegar a producir hipoxemia, disnea, claudicación respiratoria y shock séptico.

Entre las causas adquiridas se pueden señalar las infecciosas, como neumonía necrotizante, tuberculosis, parotiditis, tosferina y otras; anomalías inmunológicas, como HIV, agamaglobulinemia, granulomatosis crónica, deficiencia de IgG; reumatológicas, como el síndrome de Sjogren y la artritis reumatoide; enfermedades crónicas, como la fibrosis quística, el síndrome de Kartagener, la deficiencia de uno alfa antitripsina, el síndrome de disquinesia ciliar, el síndrome de Young; sarcoidosis y otras. Más recientemente, el reflujo gastroesofágico se ha propuesto como causa de bronquiectasias, y esto debido a la broncoaspiración de material digestivo, que es altamente irritante para la mucosa bronquial y causa de procesos inflamatorios e infecciosos recurrentes ${ }^{3}$ En un $40 \%$ de los casos no se llega a determinar el factor causal de las bronquiectasias. ${ }^{4}$

Por su morfología, se pueden clasificar como cilíndricas, saculares o varicosas, que es una combinación de las dos primeras. ${ }^{5}$ Desde el punto de vista quirúrgico, la forma más adecuada de clasificarlas es en localizadas o difusas. Esto porque la mejor posibilidad de ayudar al paciente con bronquiectasias es extirpando toda el área de tejido anormal, lo que resulta difícil y muchas veces imposible, cuando la enfermedad es difusa.
La radiografía del tórax suele mostrar áreas de infiltrado o fibrosis, como también complicaciones como bonconeumonía, derrame pleural y abscesos pulmonares. En el pasado reciente el diagnóstico se confirmaba al observar las dilataciones bronquiales en la broncografía de pacientes con historia de tos crónica, expectoración abundante de material mucopurulento $\mathrm{e}$ infecciones bronquiales a repetición. En la actualidad, la TAC de alta definición ha sustituido el uso del medio de contraste y es apropiada para valorar tanto el área específica, como el resto del pulmón, para descartar otros sitios afectados.

La manifestación clínica más frecuente es la tos crónica con expectoración mucopurulenta. Ambas, pero sobre todo la tos, pueden estar presentes muchos años antes de que se sospeche y se confirme el diagnóstico. ${ }^{6,7}$ Otros síntomas frecuentes son el cansancio y debilitamiento producto de la tos crónica y los procesos infecciosos. La obstrucción bronquial por engrosamiento de la pared bronquial en algunas áreas y el cúmulo de secreciones, son causa de disnea. Los procesos inflamatorios repetidos pueden irritar la pleura parietal y causar dolor pleurítico, y erosionar o ulcerar la mucosa, y manifestarse por hemoptisis que puede apenas teñir el esputo o ser copiosa y poner en riesgo la vida del paciente. ${ }^{8}$ Las infecciones repetidas producen destrucción del parénquima pulmonar y fibrosis, que se traducen en una disminución de la capacidad pulmonar.

Muchos pacientes con bronquiectasias que desde la infancia presentan crisis de tos con sibilancias, causadas por la inflamación bronquial y las secreciones, son catalogados y tratados como asmáticos, y en los que se manifiestan en edad adulta, como EPOC. ${ }^{4}$

El tratamiento tradicionalmente se ha hecho con broncodilatadores y antibióticos dirigidos contra Haemophilus influenza y pseudomonas, siendo esta última un marcador de la severidad de la enfermedad, aunque no un promotor de esta. ${ }^{9}$ 
Debido a que el factor clave es la acumulación de moco, que funciona como un medio de cultivo para las infecciones, el tratamiento más importante es la prevención de estas con medidas mecánicas. La destrucción del aparato ciliar y la deformidad bronquial favorece el cúmulo de secreciones que deben ser movilizadas y expectoradas. La inspirometría, la percusión, la vibración y el drenaje postural permiten la movilización y la hidratación, y el uso de mucolíticos facilita su aclaramiento. En el caso de la fibrosis quística, se ha propuesto que la inhalación de suero salino hipertónico aumenta el movimiento ciliar $\mathrm{y}$ aclaración de secreciones. ${ }^{10,11}$

El objetivo de este trabajo fue evaluar la indicación y utilidad de la cirugía en el tratamiento de pacientes con bronquiectasias.

\section{Métodos}

Se revisaron los expedientes clínicos de 36 pacientes intervenidos por bronquiectasias en el servicio de Cirugía de Tórax del Hospital Dr. Rafael Ángel Calderón Guardia (HCG), en un periodo de 13 años, entre 1996 y 2009. Se diseñó una base de datos con el fin de obtener y analizar información relativa a las características generales de los pacientes; la presentación clínica, radiológica y endoscópica; los estudios de función respiratoria; la indicación para cirugía, los resultados y complicaciones de la cirugía, así como la evolución postoperatoria.

\section{Resultados}

Durante el periodo de trece años, comprendido entre 1996 y 2009, se intervinieron 36 pacientes por bronquiectasias en el Servicio de Cirugía de Tórax del HCG. Ocho de los 36 pacientes fueron intervenidos en un segundo tiempo por enfermedad contralateral, para un total de 44 intervenciones por esta patología.

La edad en la que se operaron osciló entre los 17 y los 75 años, con promedio de 39. De ellos, 33 fueron mujeres y 3 hombres, con relación de 11 a 1 .

En 10 casos las bronquiectasias fueron secundarias a secuelas de tuberculosis; en uno, la paciente sufría del síndrome de Kartagener; un paciente tenía historia se reflujo gastroesofágico severo y, posteriormente, inició los síntomas respiratorios; un caso con secuelas de una infección respiratoria en la que se confirmó $\mathrm{H} 1 \mathrm{~N} 1$, y en 23 no fue posible establecer con precisión la causa primaria, por falta de estudios e información documentada. Algunos pacientes tenían antecedente de bronconeumonía, pero no fue posible definir si fue la causa o consecuencia de las bronquiectasias.

Diecinueve pacientes tuvieron ingresos previos relacionados: 10 por tuberculosis, 6 por infecciones respiratorias y 3 por bronconeumonía.

Los síntomas y signos documentados se muestran en el Cuadro1, siendo que todos tenían historia de tos crónica y expectoración verdosa o amarillenta; en 8 pacientes desde la infancia y en otros de duración variable. En 29 se planteó el diagnóstico de asma por la presencia de estertores y sibilancias; en algunos desde la infancia y con historia familiar, pero en 10 no se documentó ningún antecedente familiar ni historia de asma en la infancia. Solo 3 pacientes tenían el antecedente de tabaquismo.

En la radiografía del tórax se describen diversidad de hallazgos, tales como: infiltrados pulmonares en 13 casos, áreas de fibrosis en 10, infiltrados nodulares en 4, áreas quísticas en 2, atelectasia segmentaria en 1, y solo en un caso se describe bronquiectasias.

En la TAC de tórax se describe la presencia de bronquiectasias en 32 pacientes (en 6 quísticas, en 4 cilíndricas, en 4 más fibrosis y en 4 más infiltrados), y en 4 casos lo que se describe es la presencia de áreas fibróticas más infiltrados nodulares. Las bronquiectasias se clasificaron como localizadas en 16 casos, localizadas bilaterales en 13, difusas en 3 y difusas bilaterales en 4 .

La localización fue de 27 del lado derecho y 17 del izquierdo. En el Cuadro 2 se muestra la localización por lóbulos, siendo el lóbulo medio el más afectado.

\section{Cuadro 1. Signos y síntomas de 36 pacientes intervenidos por bronquiectasias en el Hospital Dr. R. Á. Calderón Guardia}

\begin{tabular}{|lc|}
\hline \multicolumn{1}{|c|}{ Signos y síntomas } & Número \\
\hline Tos crónica & 36 \\
Expectoración purulenta & 36 \\
Sibilancias & 34 \\
Estertores & 31 \\
Fiebre & 31 \\
Afección del estado general & 27 \\
Dolor torácico & 19 \\
Hemoptisis & 11 \\
\hline
\end{tabular}

Cuadro 2. Localización de las bronquiectasias en 44 cirugías realizadas en el Hospital Dr. R. Á. Calderón Guardia

\begin{tabular}{|lcc|}
\hline \multicolumn{1}{|c}{ Lóbulo } & Número & Porcentaje \\
\hline Superior derecho & 7 & $16 \%$ \\
Medio & 18 & $41 \%$ \\
Inferior derecho & 5 & $11 \%$ \\
Superior izquierdo & 10 & $23 \%$ \\
Inferior izquierdo & 4 & $9 \%$ \\
\hline
\end{tabular}




\begin{tabular}{|lc|}
\hline \multicolumn{2}{|c|}{$\begin{array}{c}\text { Cuadro 3. Cirugía realizada en } \\
\text { intervenciones por bronquiectasias }\end{array}$} \\
\hline \multicolumn{1}{|c|}{ Intervención quirúrgica } & Número \\
\hline Lobectomía & 23 \\
Resección en cuña & 10 \\
Segmentectomía & 7 \\
Lobectomía más segmentectomía & 2 \\
Resección en cuña por toracoscopía & 1 \\
Segmentectomía por VATS & 1 \\
\hline
\end{tabular}

Las pruebas de función respiratoria se realizaron a los 35 pacientes y resultaron normales en 5 , con obstrucción leve en 11 , moderada en 8 , severa en 7 , y con obstrucción moderada más restricción en 4 .

En 25 pacientes se realizó broncoscopia y en todos se describe la presencia de abundantes secreciones purulentas, $\mathrm{y}$ en ninguno el hallazgo de alteraciones anatómicas u otros.

La cirugía realizada en las 44 intervenciones se muestra en el Cuadro 3, siendo que por la extensión de la enfermedad, en 23 casos fue necesario realizar lobectomía. En 15 hojas operatorias se describe el hallazgo de múltiples adherencias pleurales, más firmes en los casos con antecedente de tuberculosis. En 12 casos se describe la presencia de un área muy localizada de enfermedad con el resto del pulmón de aspecto normal, y esto incluye los dos casos realizados por toracoscopia. La descripción macroscópica del área comprometida es la presencia de un área del pulmón con fibrosis, retracción atelectásica, y donde se palpan pequeños nódulos que corresponden a los bronquios dilatados, inflamados y llenos de material purulento. En todos los casos recientes se utilizó un tubo endotraqueal de doble lumen para facilitar la cirugía, pero también para evitar el desplazamiento del material purulento hacia el pulmón contralateral.

La estancia postoperatoria fue de 3 días en 3 pacientes, 4 días en 12, 5 días en 15, entre 7 y 17 días en 12 pacientes y 25 días en 2 . La estancia mayor a 7 días obedeció a complicaciones, siendo la infección respiratoria con producción de secreciones y fiebre la más frecuente, y se presentó en 5 casos; 4 pacientes sufrieron bronconeumonía, con necesidad de ventilación mecánica de los dos que duraron 25 días hospitalizados; 2 pacientes presentaron sepsis de la herida quirúrgica; 1 caso se complicó con empiema que requirió decorticación por toracoscopia; 1 caso de neumotórax persistente y 1 paciente presentó una fístula de líquido céfaloraquídeo en el sitio de inserción del catéter peridural para analgesia. No se produjo ningún caso de mortalidad quirúrgica.

En el momento de revisar los expedientes, 8 pacientes habían fallecido por causas diversas, 5 con complicaciones pulmonares. En relación con la cirugía y de acuerdo con la última anotación en el expediente, 12 pacientes fueron dados de alta en buenas condiciones, para 6 se describe que se encontraban bien y seguían en control, 6 continuaban con tos productiva, 8 en condición regular con infecciones respiratorias recurrentes, 2 en mala condición con infecciones frecuentes, y 2 habían requerido internamientos por infecciones respiratorias severas postoperatorias.

Con el fin de identificar algún factor pronóstico de la cirugía, se relacionó la evolución postoperatoria con la extensión de la enfermedad y, tal como se muestra en el Cuadro 4, no se encontró una relación proporcional predictiva.

\section{Discusión}

Las bronquiectasias, aunque puede plantearse la posibilidad de que sean una anormalidad bronquial congénita, en la gran mayoría de los casos son secundarias a una enfermedad primaria infecciosa, inflamatoria oinmunológica, por lo que debe mejorarse el estudio de los pacientes con el fin de establecer la causa.

$\mathrm{La}$ presencia de tos crónica y expectoración mucopurulenta crónicas debe hacer sospechar la presencia de bronquiectasias y no de asma, sobre todo sin una historia familiar. El hallazgo de lesiones en la radiografía del tórax en pacientes con dicha historia clínica, debe complementarse con una TAC con protocolo para bronquiectasias.

\begin{tabular}{|lcl|}
\hline \multicolumn{2}{|c|}{ Cuadro 4 Relación entre estado postoperatorio y la extensión de las bronquiectasias } \\
en 36 pacientes operados
\end{tabular}


Debido a la irreversibilidad de las lesiones, el paciente debe de ser muy bien informado e ilustrado sobre su problema, para que aprenda a expulsar las secreciones acumuladas y así evitar o espaciar los cuadros infecciosos.

Dado que la anatomía y fisiología pulmonar en ambos géneros es igual, la posible explicación para la mayor incidencia en mujeres, es el recato para expulsar las secreciones, por lo que se debe enfatizar la importancia de hacerlo.

La cirugía sigue siendo una alternativa, sobre todo cuando el tratamiento médico falla en proporcionar una calidad de vida aceptable. Con el fin de establecer mejor las indicaciones de cirugía y el pronóstico, deben realizarse más estudios.

\section{Referencias}

1. Laennec RTH. A treatise of the disease of the chest. Forbes $J$ translator. New York : Library of New York Academy of Medicine, Hafner Publishing, 1962 p. 78.

2. Reid LM. Reduction in bronchial subdivision in bronchial bronchiectasis. Thorax 1950; 5: 233-247
3. Llowite J, Spiegler P, Chawla S. Bronchiectasis: new findings in the pathogenesis and treatment of this disease. Curr Opin Infect Dis 2008; 21:163-167.

4. Cohen M, Sahn S. Bronchiectasis in systemic diseases. Chest 1999; 116:1063-1074

5. Whitwell F. A study of the pathology and pathogenesis of bronchiectasis. Thorax 1952; 7: 213-239.

6. King PT, Holdsworth SR, Freezer NI. Characterization of the onset and presenting clinical features of the adult bronchiectasis. Resp. Med. 2006; 100:2183-2189.

7. Baker AF. Bronchiectasis. N Engl J of Med 2002; 346: 1383-1393.

8. Nicota MB, Rivera M, Dale AM, Shepherd R, Carter R. Clinical pathophysiologic and microbiologic characterization of bronchiectasis in aging cohort. Chest 1995; 108:955-961.

9. Angrill J. Augusti C, de Celi R, Rañó A, González J, Solé T, Xaubert A, Rodriguez R. Bacterial colonisation in patients with bronchiectasis: microbiological pattern and risk factors. Thorax 2002; 57:15-19.

10. Elkins MR, Robinson M, Rose BR. Harbour C, Moriarty CP, Marks GB, Belousova EG, Xuan W, Bye P. A controlled trial of long term inhaled hypertonic in patients with cystic fibrosis. N Engl J of Med 2008; 354:229-240.

11. Thompson RM, Armstrong JG, Looke DF. Gastroesophageal reflux disease, acid suppression and Mycobacterium avium complex pulmonary disease. Chest 2007; 131:1166-1172.

\section{Comité Editorial Acta Médica Costarricense}

\section{Lista de revisión para autores}

Por favor, complete esta lista antes de enviar su manuscrito.

$\square$ Carta de presentación

$\square$ Tres copias completas del manuscrito con cuadros y figuras, y en formato digital

Página de título

$\square$ Título

$\square$ Título corto, no mayor de 45 caracteres

$\square$ Autor(es) y afiliación(es)

$\square$ Lugar donde se realizó el trabajo

$\square$ Descriptores en español e inglés

$\square$ Abreviaturas

$\square$ Dirección, teléfono y número de fax del autor a quien se debe dirigir toda correspondencia

$\square$ Fuentes de apoyo y manifestación de conflictos de interés

\section{Artículo (a doble espacio)}

$\square$ Carta de aprobación del estudio por Comité Ético - científico

$\square$ Resumen estructurado en español

$\square$ Resumen estructurado en inglés

$\square$ Introducción

$\square$ Materiales y métodos

$\square$ Resultados

$\square$ Discusión

$\square$ Agradecimientos y colaboradores

$\square$ Referencias

$\square$ Cuadros, leyendas de las figuras, figuras

$\square$ Permiso para reproducir todo material previamente publicado y autorización de pacientes para publicar fotografías

$\square$ Carta de cada autor de asignación de derechos, responsabilidad de participación y declaración de financiamiento, si existiera (puede ser una, firmada por todos los autores).

La Revista Acta Médica Costarricense les informa que para 2011 los médicos interesados en recibir el ejemplar impreso a su dirección geográfica o apartado postal, deben solicitarlo por escrito a: actamedica@medicos.cr, anotando los siguientes datos:

Nombre completo:

Código:

Teléfono de habitación:

Dirección geográfica o apartado: 